\title{
MMP-7 and MMP-9 are overexpressed in the synovial tissue from severe temporomandibular joint dysfunction
}

\author{
Carla Loreto, ${ }^{1}$ Veronica Filetti, ${ }^{1}$ Luis Eduardo Almeida, ${ }^{2}$ Giusy Rita Maria La Rosa, ${ }^{3}$ Rosalia Leonardi, ${ }^{3}$ \\ Cristina Grippaudo, ${ }^{4}$ Antonino Lo Giudice ${ }^{3}$ \\ ${ }^{1}$ Department of Biomedical and Biotechnological Sciences, Section of Anatomy, University of Catania, Italy \\ ${ }^{2}$ School of Dentistry, Marquette University, Milwaukee, WI, USA \\ ${ }^{3}$ Department of Orthodontics, School of Dentistry, University of Catania, Italy \\ ${ }^{4}$ Department of Orthodontics, School of Dentistry, Catholic University of the Sacred Heart, Rome, Italy
}

\begin{abstract}
Matrix metalloproteinases (MMPs) are tissue-enzymes that play a key role during the remodeling process, such as in inflammatory diseases. MMP-7 and MMP-9 have been shown to be implicated in extracellular matrix homeostasis and in joint disc remodeling. The objective of this study was to determine the relation of MMP-7 and MMP-9 expression with severe temporomandibular joint dysfunction, in particular with anterior disk displacement without reduction (ADDwoR), using an immunohistochemical approach. Therefore, twenty human temporomandibular synovia in the test group and ten in the control group were collected. The results showed there was a statistically significant difference $(\mathrm{P}<0.001)$ for morphometric and densitometric analysis of both detected MMPs in control and test groups. In conclusion, MMP-7 and MMP-9 were overexpressed in the synovial tissue of patients with ADDwoR.
\end{abstract}

Key words: MMP-7; MMP-9; TMJ synovial; TMJ dysfunction.

Correspondence: Veronica Filetti, Department BIOMETEC, Section of Anatomy, University of Catania, via S. Sofia 87, 95123 Catania, Italy. E-mail: verofiletti@gmail.com

Contributions: CL, LR, contribution to design experiments and data acquisition, manuscript drafting; GRMLR, LGA, contribution to study design and data acquisition; VF, LEA, CG, contribution to study design, manuscript critical revision. All authors read and approved the final manuscript.

Conflict of interest: The authors report no conflicts of interest related to this study.

Ethics approval and consent to participate: The Ethics Committee of the Pontifical Catholic University of Paranà, according to Resolution 196/96 of the National Health Council (registration number 104), approved the study.

Patient consent for publication: Not applicable.

Informed consent: Patients' informed consent had been obtained before tissue collection. 


\section{Introduction}

Temporomandibular disorders (TMDs) define a number of clinical problems involving masticatory musculature, temporomandibular joint (TMJ), and associated structures. Many TMDs are characterized by similar symptoms described as pain in the joint, noisy interferences during mastication, and movement restrictions.

Several studies have investigated the changes in the composition of the articular TMJ disc for understanding the significance of the pathological process of internal derangement (ID) using immunohistochemical approaches..$^{2-5}$

A growing body of evidence indicates that matrix metalloproteinases (MMPs) are associated with a family of zinc-dependent endopeptidases, responsible for extracellular matrix (ECM) protein degradation by cleavage of internal peptide bonds. ${ }^{6,7}$ They are involved in physiological tissue remodeling, but in some conditions the overexpression of MMPs result in tissue destruction. ${ }^{8}$

Indeed, the upregulation of some MMPs has been observed in the TMJ synovial fluid pathological conditions, resulting the primary enzymes involved in matrix turnover. ${ }^{9-14}$

Studies about TMJ disc analyzing the synovial fluid (SF) of patients with IDs have shown a relationship between MMPs overexpression and TMJ degeneration, indicating a possible marker for these pathologies. ${ }^{8-10,15-18}$ In particular, MMP-3 is involved in the pathological destruction process of TMJ osteoarthritis combined with disc displacement. ${ }^{16}$ High levels of MMP-1, MMP-8, and MMP-13, MMP-2 and MMP-9 have been reported in the TMJ-SF of patients with ID. ${ }^{8,17}$ Furthermore, MMP-3 and MMP-7 were correlated with bone apposition. ${ }^{18}$

MMP-7 and MMP-9 have been shown to play an important role in ECM homeostasis and in joint disc remodeling. ${ }^{15}$ An MMP9 increase has been observed in the serum and SF of arthritic patients and it is considered a marker of arthritis and cartilage degradation in animal studies..$^{10}$ MMP-9 was highly expressed in patients with anterior disk displacement without reduction (ADDwoR) and advanced osteoarthritis. ${ }^{19-22}$

To date, to our best knowledge, no studies have evaluated the MMPs expression in the synovial tissue. It is reasonable to think that the presence of MMPs in the SF derives from the synovial tissue. Therefore, the aim of this study was to assess the expression of MMP-7 and MMP-9 in the synovial tissue of patients with a severe TMJ dysfunction.

\section{Materials and Methods}

\section{Patients and tissues}

Twenty paraffin blocks of displaced TMJ synovia specimens were retrieved from the archives of the Pontifical Catholic University of Paranà (Brazil). ${ }^{11,23,24}$ Their use had already been approved by the ethics committee of the Pontifical Catholic University of Paranà according to Resolution 196/96 of the National Health Council (registration number 104); similarly, the patients' informed consent had been obtained before tissue collection. The specimens had been removed from eleven females and nine males with ADDwoR diagnosed on the basis of history, clinical examination, and magnetic resonance imaging data. The diagnosis for disc excision was ID with pain and functional impairment.

The inclusion criteria were: i) unsuccessful conservative management; ii) tenderness to TMJ palpation; iii) TMJ pain or interference with jaw movement. The exclusion criteria were: i) other TMJ diseases; ii) dento-facial deformity; iii) major jaw trauma; iv) previous TMJ surgery; v) prior TMJ treatment with steroid injections. The discs removed from the twenty patients were macroscopically deformed and none had preserved a normal biconcave shape.

Ten TMJ synovia specimens from the collection of Catania Human Anatomy Institute (Italy) were analyzed as controls. They were autopsy specimens from five male donors and five female donors which were selected for their virtually normal shape and condition, since none had macroscopic signs of degenerative or inflammatory joint disease on dissection, nor were they displaced; the donors' clinical histories were negative for generalized joint disease or TMJ arthropathy.

\section{Histology}

The TMJ synovia sections were rinsed in phosphate buffered saline (PBS; Sigma, Milan, Italy), fixed in 10\% buffered formalin, as previously described. ${ }^{25,26}$ After an overnight wash, specimens were dehydrated in graded ethanol, cleared in xylene and paraffinembedded, preserving their anatomical orientation. Sections (4-5 $\mu \mathrm{m}$ in thickness) were cut from paraffin blocks using a microtome, mounted on silane-coated slides (Dako, Glostrup, Denmark) and stored at room temperature. The sections were then stained with hematoxylin and eosin and observed using a Zeiss Axioplan light microscope (Carl Zeiss, Oberkochen, Germany) for general morphology. Finally, representative photomicrographs were captured using a Zeiss AxioCam MRc5 digital camera (Carl Zeiss).

\section{Immunohistochemistry}

Samples were fixed in $10 \%$ buffered formalin for $2 \mathrm{~h}$; after an overnight wash, specimens were dehydrated in graded ethanol and paraffin-embedded. Sections (3-4 $\mu \mathrm{m}$ in thickness) were cut, mounted on silane-coated slides, and air-dried. For immunohistochemical analysis, specimens were processed as previously described. ${ }^{27}$ Briefly, the slides were dewaxed in xylene, re-hydrated with graded ethanol, incubated for $30 \mathrm{~min}$ in $0.3 \%$ $\mathrm{H}_{2} \mathrm{O}_{2} /$ methanol solution to quench endogenous peroxidase activity, and then rinsed for 20 min with PBS. Antigen retrieval was performed using a microwave oven $(750 \mathrm{~W})(5 \mathrm{~min} \times 3)$ in capped polypropylene slide-holders with citrate buffer $(10 \mathrm{mM}$ citric acid, $0.05 \%$ Tween 20, pH 6.0; Bio-Optica, Milan, Italy). The sections were incubated overnight at $4^{\circ} \mathrm{C}$ with anti-MMP-7 or anti-MMP-9 monoclonal antibodies (sc-80205 and sc-13520 respectively, Santa Cruz Biotechnology, Inc., Dallas, TX, USA), both diluted 1:100 in PBS. ${ }^{15}$ Immune complexes were then treated with a biotinylated link antibody and then detected with peroxidase labeled streptavin (LSAB+ System-HRP, K0690; Dako), both incubated for $10 \mathrm{~min}$ at room temperature. The immunoreaction was visualized by 3,3 'diaminobenzidine and $0.02 \%$ hydrogen peroxide solution (DAB substrate Chromogen System; Dako), as previously described. ${ }^{27}$ The sections were lightly counterstained with Mayer's hematoxylin (Histolab Products AB, Göteborg, Sweden) mounted in GVA (Zymed Laboratories, San Francisco, CA, USA). The sections were observed and photographed as described above.

\section{Evaluation of immunohistochemistry}

The MMP-7 and MMP-9 antibody-staining status were identified as either negative or positive. Immunohistochemical positive staining was defined by the presence of brown chromogen detection on the edge of the hematoxylin-stained cell nucleus, distributed within the cytoplasm or in the membrane via evaluation by light microscopy as previously described. ${ }^{28}$ Positive controls consisted of tissue specimens with known antigenic positivity. Sections treated with PBS without the primary antibodies served as negative controls. Seven fields of about $600,000 \mu \mathrm{m}^{2}$, randomly selected from each section, were considered for morphometric and 
densitometric analysis. The percentage areas (morphometric analysis) stained with MMP-7 and MMP-9 antibodies were expressed as $\%$ positive, dark brown pixels of the analyzed fields. While, the levels (high/low) of staining intensity of positive areas (densitometric analysis) were expressed as densitometric count $\left(\mathrm{pixel}^{2}\right)$ of positive, dark brown pixels of the analyzed fields. These parameters were calculated using software for image acquisition (AxioVision Release 4.8.2 - SP2 Software, Carl Zeiss Microscopy $\mathrm{GmbH}$, Jena, Germany). Data were expressed as mean \pm standard deviation (SD). Digital micrographs were taken and fitted as previously described.

\section{Statistical analysis}

Statistical analysis was performed using GraphPad Prism 7.0 (GraphPad Software, Inc., La Jolla, CA, USA). Data were tested for normality with the Kolmogorov-Smirnov test. All variables were normally distributed. Student's $t$-test was used for comparisons between two means. P-values less than $0.05(\mathrm{P}<0.05)$ and $0.001(\mathrm{P}<0.001)$ were considered statistically and very statistically significant, respectively.

\section{Results}

MMP-7 and MMP-9 expression was confirmed, following immunohistochemistry. Staining was localized in fibroblast-like type B cells expressing MMP-7 and MMP-9. All experimental samples were identified as positively stained.

As shown in Figure 1, densitometric expression of MMP-7 and MMP-9 was significantly increased in ADDwoR when compared to the controls $(\mathrm{P}<0.001)$. However, as shown in Figure 2, there was no significant difference between MMP-7 (Figure 2A) and MMP-9 (Figure 2B) immunostainings ( $\mathrm{P}>0.05$ ). ADDwoR fibroblasts staining intensity, localized in the inner layer of the synovial membrane, was statistically significant compared to the control tissue (Figure 2C) $(\mathrm{P}<0.001)$.

\section{Discussion}

MMPs have been shown to play an important role in ECM homeostasis and in joint disc remodelling. Our results showed a statistically significant difference in MMP-7 and MMP-9 immuno-

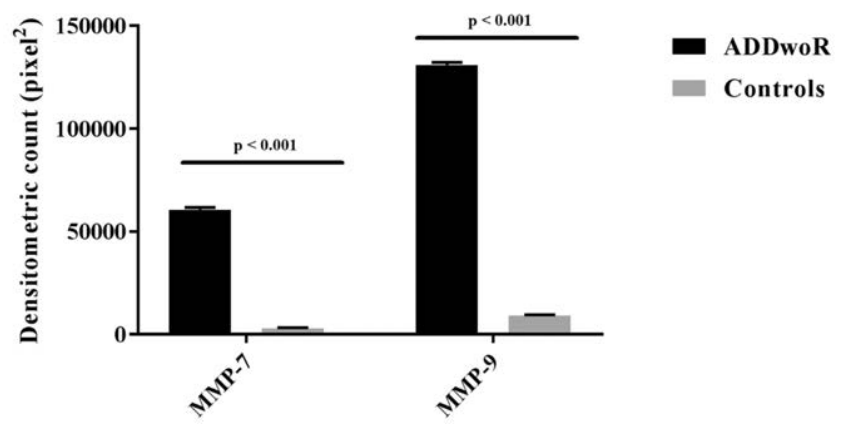

Figure 1. Densitometric analysis. A bar chart representing a comparison of the percentage of MMP-7 and MMP-9 immunostained area in ADDwo Rynovial tissues vs. synovial control tissues, expressed by positive percentage, dark brown pixels of the analyzed fields. Data are presented as mean $\pm S D$. ${ }^{*} \mathrm{P}<0.001$. expression was detected between the synovial tissues of ADDwoR and control samples. The expression of these MMPs is regulated by several factors including a variety of cytokines, which play an important role in TMJ ID pathogenesis. They have indeed been demonstrated in SF of pathological TMJ, suggesting that their expression could be a potential biochemical marker for articular cartilage degradation..$^{8,15,22}$
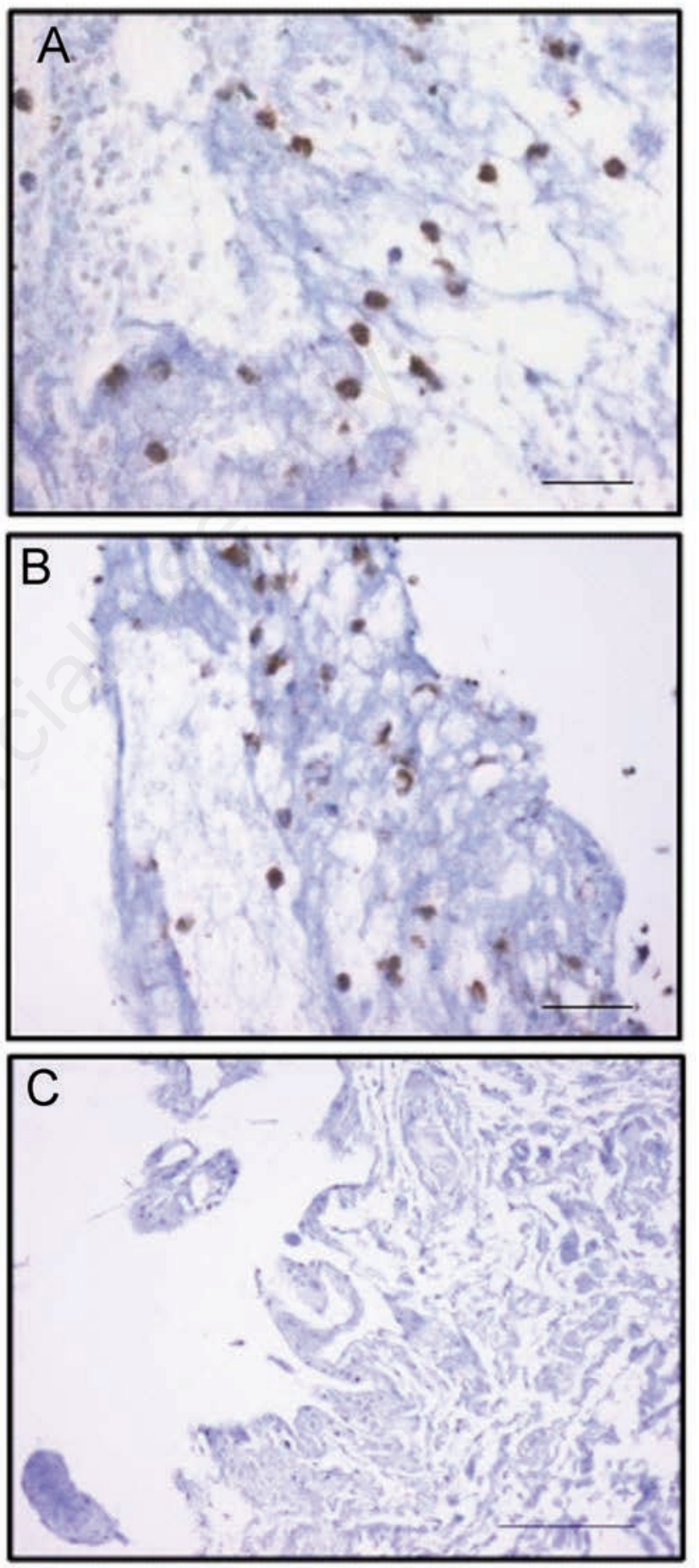

Figure 2. MMP-7 (A) and MMP-9 (B) immunoexpression of fibroblasts in synovial tissue sample of ADDwoR patient, respectively; magnification $600 \mathrm{x}$; scale bars: $30 \mu \mathrm{m}$; ${ }^{*} \mathrm{P}>\mathbf{0 . 0 5}$. C) MMP7 immunoexpression in synovial tissue control sample; magnification $400 \mathrm{x}$; scale bar: $60 \mu \mathrm{m}$. 
MMP-7 and MMP-9 are expressed in arthritic joints and can degrade a number of matrix proteins in the joint. ${ }^{29}$ In osteoarthritis, synovial macrophages, synovial fibroblasts, and chondrocytes may induce the release of MMPs which destroy joint cartilage. ${ }^{11,30}$ In particular, human TMJ synovial cells have been reported to synthesize MMP-1, MMP-3, and MMP-9 in vitro. ${ }^{31,32}$

Transmission electron microscopy analysis showed two types of synovial lining cells, including the macrophages-like type A and fibroblast-like type B cells in the synovial lining layer of TMJ. In particular, a secretory function was attributed to fibroblast-like type B cells. ${ }^{29}$ These cells secrete type I and II collagens, fibronectin, and glycosaminoglycans into the synovial interstitium and fluids..$^{29,33-35}$ Therefore, it is reasonable to think that the MMPs overexpression in the synovial fluid derives from the secretory activity of fibroblast-like type B cells that showed in our study an overexpression of both MMP-7 and MMP-9.

In conclusion, within the limits of the present study, MMP-7 and MMP-9 were demonstrated to be overexpressed in the synovial tissue of patients with ADDwoR. Further studies about this topic may contribute to better understand the role of the inflammation process on tissue remodeling and destruction in the TMJ.

\section{References}

1. Stegenga B. Temporomandibular joint degenerative diseases: Clinical diagnosis. In: Stegenga B, de Bont LGM, editors. Management of temporomandibular joint degenerative diseases. Basel: Birkhauser; 1996.

2. Matsumoto $T$, Inayama M, Tojyo I, Kiga N, Fujita S. Expression of hyaluronan synthase 3 in deformed human temporomandibular joint discs: in vivo and in vitro studies. Eur J Histochem 2010;54:e50. doi: 10.4081/ejh.2010.e50.

3. Kiga N, Tojyo I, Matsumoto T, Hiraishi Y, Shinohara Y, Makino S, et al. Expression of lumican and fibromodulin following interleukin-1 beta stimulation of disc cells of the human temporomandibular joint. Eur J Histochem 2011;55: e11. doi: 10.4081/ejh.2011.e11.

4. Okamoto K, Kiga N, Shinohara Y, Tojyo I, Fujita S. Effect of interleukin-1beta and dehydroepiandrosterone on the expression of lumican and fibromodulin in fibroblast-like synovial cells of the human temporomandibular joint. Eur J Histochem 2015;59:2440. doi: 10.4081/ejh.2015.2440.

5. Loreto C, Almeida LE, Migliore MR, Caltabiano M, Leonardi R. TRAIL, DR5 and caspase 3-dependent apoptosis in vessels of diseased human temporomandibular joint disc. An immunohistochemical study. Eur J Histochem 2010;54:e40. doi: 10. 4081/ejh.2010.e40.

6. Hopps E, Caimi G. Matrix metalloproteases as a pharmacological target in cardiovascular diseases. Eur Rev Med Pharmacol Sci 2015;19:2583-89.

7. Ferreira LM, Moura ÁF, Barbosa GA, Pereira HS, Calderon PS. Do matrix metalloproteinases play a role in degenerative disease of temporomandibular joint? A systematic review. Cranio 2016;34:112-7.

8. Srinivas R, Sorsa T, Tjaderhane L, Niemi E, Raustia A, Pernu H, et al. Matrix metalloproteinases in mild and severe temporomandibular joint internal derangement synovial fluid. Oral Surg Oral Med Oral Pathol Oral Radiol Endod 2001;91:517-25.

9. Leonardi R, Loreto C, Barbato E, Caltabiano R, Lombardo C, Musumeci G, et al. MMP-13 (collagenase 3) localization in human temporomandibular joint discs with internal derangement. Acta Histochem 2008;110:314-18.

10. Ishimaru JI, Oguma Y, Goss AN. Matrix metalloproteinase and tissue inhibitor of metalloproteinase in serum and lavage syn- ovial fluid of patients with temporomandibular joint disorders. Br J Oral Maxillofac Surg 2000;38:354-59.

11. Leonardi R, Almeida LE, Loreto C. Lubricin immunohistochemical expression in human temporomandibular joint disc with internal derangement. J Oral Pathol Med 2011;40:587-92.

12. Kubota E, Kubota T, Matsumoto J, Shibata T, Murakami KI. Synovial fluid cytokines and proteinases as markers of temporomandibular joint disease. J Oral Maxillofac Surg 1998;56: 192-8.

13. Kubota E, Imamura H, Kubota T, Shibata T, Murakami K. Interleukin $1 \mathrm{~b}$ and stromelysin (MMP3) activity of synovial fluid as possible markers of osteoarthritis in the temporomandibular joint. J Oral Maxillofac Surg 1997;55:20-7.

14. Loreto C, Lombardo C, Caltabiano R, Filetti V, Vitale E, Seminara D, et al. Immunohistochemical expression and localization of MMP-9, MMP-13, E-Cadherin and Ki-67 in road pavers' skin chronically exposed to bitumen products. Histol Histopathol 2019;34:1141-50.

15. Loreto C, Leonardi R, Musumeci G, Pannone G, Castorina S. An ex vivo study on immunohistochemical localization of MMP-7 and MMP-9 in temporomandibular joint discs with internal derangement. Eur J Histochem 2013;57:e12. doi: 10.4081/ejh.2013.e12.

16. Jiang Q, Qiu YT, Chen MJ, Zhang ZY, Yang C. Synovial TGFbeta1 and MMP-3 levels and their correlation with the progression of temporomandibular joint osteoarthritis combined with disc displacement: A preliminary study. Biomed Rep 2013;1: 218-22.

17. Mizui T, Ishimaru J, Miyamoto K, Kurita K. Matrix metalloproteinase-2 in synovial lavage fluid of patients with disorders of the temporomandibular joint. Br J Oral Maxillofac Surg 2001;39:310-4.

18. Cevidanes LH, Walker D, Schilling J, Sugai J, Giannobile W, Paniagua B, et al. 3D osteoarthritic changes in TMJ condylar morphology correlates with specific systemic and local biomarkers of disease. Osteoarthritis Cartilage 2014;22:1657-67.

19. Edelstein C. Biomarkers of kidney disease, 2nd ed. Amsterdm; Elsevier: 2017.

20. Nagase H, Woessner JF Jr. Matrix metalloproteinases. J Biol Chem 1999;274:21491-4.

21. Makowski GS, Ramsby ML. Autoactivation profiles of calcium-dependent matrix metalloproteinase-2 and -9 in inflammatory synovial fluid: effect of pyrophosphate and bisphosphonates. Clin Chim Acta 2005;358:182-91.

22. Yoshida K, Takatsuka S, Hatada E, Nakamura H, Tanaka A, Ueki K, et al. Expression of matrix metalloproteinases and aggrecanase in the synovial fluids of patients with symptomatic temporomandibular disorders. Oral Surg Oral Med Oral Pathol Oral Radiol Endod 2006;102:22-7.

23. Loreto C, Galanti C, Almeida LE, Leonardi R, Pannone G, Musumeci G, et al. Expression and localization of aquaporin1 in temporomandibular joint disc with internal derangement. J Oral Pathol Med 2012;41:642-47.

24. Sicurezza E, Loreto C, Musumeci G, Almeida LE, Rusu M, Grasso $C$, et al. Expression of $\beta$-defensin 4 on temporomandibular joint discs with anterior displacement without reduction. J Craniomaxillofac Surg 2013;57:e12.

25. Leonardi R, Loreto C, Barbato E, Polimeni A, Caltabiano R, Lo Muzio L. A histochemical survey of the human temporomandibular joint disc of patients with internal derangement without reduction. J Craniofac Surg 2007;18:1429-33.

26. Castorina S, Lombardo C, Castrogiovanni P, Musumeci G, Barbato E, Almeida LE, Leonardi R. P53 and VEGF expression in human temporomandibular joint discs with derangement correlate with degeneration. J Biol Regul Homeost 
Agents 2019;33:1657-62.

27. Leonardi R, Talic NF, Loreto C. MMP-13 (collagenase 3) immunolocalisation during initial orthodontic tooth movememnt in rats. Acta Histochem 2007;109:215-20.

28. Davidson RK, Waters JG, Kevorkian L, Darrah C, Cooper A, Donell ST, et al. Expression profiling of metalloproteinases and their inhibitors in synovium and cartilage. Arthritis Res Ther 2006;8:R124

29. Nozawa-Inoue K, Amizuka N, Ikeda N, Suzuki A, Kawano Y, Maeda T. Synovial membrane in the temporomandibular joint-its morphology, function and development. Arch Histol Cytol 2003;66:289-306.

30. Musumeci G, Loreto C, Giunta S, Rapisarda V, Szychlinska MA, Imbesi R, et al. Angiogenesis correlates with macrophage and mast cell infiltration in lung tissue of animals exposed to fluoro-edenite fibers. Exp Cell Res 2016;346:91-8.

31. Kubota T, Kubota E, Matsumoto A, Kawai Y, Saito H, Mikuni-
Takagaki Y, et al. Identification of matrix metalloproteinases (MMPs) in synovial fluid from patients with temporomandibular disorder. Eur J Oral Sci 1998;106:992-8.

32. Akamine Y, Kakudo K, Kondo M, Ota K, Muroi Y, Yoshikawa $\mathrm{H}$, et al. Prolonged matrix metalloproteinase-3 high expression after cyclic compressive load on human synovial cells in threedimensional cultured tissue. Int J Oral Maxillofac Surg 2012; 41:874-81.

33. Gu Z, Feng J, Shibata T, Hu J, Zhang Z. Type II collagen and aggrecan mRNA expression by in situ hybridization in rabbit temporomandibular joint posterior attachment following disc displacement. Arch Oral Biol 2003;48:55-62.

34. Mapp PI, Revell PA. Fibronectin production by synovial intimal cells. Rheumatol Int 1985;5:229-37.

35. Roy S, Ghadially FN. Synthesis of hyaluronic acid by synovial cells. J Pathol Bacteriol 1967;93:555-7. 